\title{
Nucleon Structure from Dynamical Lattice QCD
}

\author{
Huey-Wen Lin \\ for the RBC-UKQCD Collaboration \\ Jefferson Laboaratory, 12000 Jefferson Avenue, Newport News, VA 23606 \\ RIKEN-BNL Research Center, Brookhaven National Laboratory, Upton, NY 11973
}

\begin{abstract}
We present lattice QCD numerical calculations of hadronic structure functions and form factors from full-QCD lattices, with a chirally symmetric fermion action, domain-wall fermions, for the sea and valence quarks. The lattice spacing is about $0.12 \mathrm{fm}$ with physical volume approximately $(2 \mathrm{fm})^{3}$ for RBC 2-flavor ensembles and $(3 \mathrm{fm})^{3}$ for RBC/UKQCD $2+1$-flavor dynamical ones. The lightest sea quark mass is about $1 / 2$ the strange quark mass for the former ensembles and $1 / 4$ for the latter ones. Our calculations include: isovector vector- and axial-charge form factors and the first few moments of the polarized and unpolarized structure functions of the nucleon. Nonperturbative renormalization in $\mathrm{RI} / \mathrm{MOM}$ scheme is applied.
\end{abstract}

Keywords: QCD, Lattice, Nucleon structure

PACS: 11.15.Ha, 11.30.Rd, 12.38.Aw, 12.38.-t 12.38.Gc

\section{INTRODUCTION}

Low-energy matrix elements play an important role in understanding QCD. However, conventional theory fails to calculate these quantities without recourse to nonperturbative treatments or effective theories. Lattice QCD is a discrete version of continuum QCD, which allows us to calculate nonperturtatively in QCD. Using Monte Carlo integration and "importance sampling" techniques, one can calculate the path integral directly without further assumptions. At the end of the calculation, one is required to take the lattice spacing $a \rightarrow 0$ and the volume $V \rightarrow \infty$ to reach the continuum limit.

The structure functions are measured in the deep inelastic scattering of leptons off nucleons, where the cross section can be factorized in terms of the products of leptonic $L^{\mu \nu}$ and hadronic $W^{\mu \nu}$ tensors. Since the leptonic tensor is known, the cross section provides us with information about the hadronic tensor. The hadronic part can be further divided into the symmetric, unpolarized, spin-averaged part

$$
W^{\{\mu v\}}\left(x, Q^{2}\right)=\left(-g^{\mu \nu}+\frac{q^{\mu} q^{v}}{q^{2}}\right) F_{1}\left(x, Q^{2}\right)+\left(p^{\mu}-\frac{v}{q^{2}} q^{\mu}\right)\left(p^{v}-\frac{v}{q^{2}} q^{v}\right) \frac{F_{2}\left(x, Q^{2}\right)}{v},
$$

and the anti-symmetric, polarized one

$$
W^{[\mu v]}\left(x, Q^{2}\right)=i \varepsilon^{\mu v \rho \sigma} q_{\rho}\left(\frac{s_{\sigma}}{v}\left(g_{1}\left(x, Q^{2}\right)+g_{2}\left(x, Q^{2}\right)\right)-\frac{q \cdot s p_{\sigma}}{v^{2}} g_{2}\left(x, Q^{2}\right)\right),
$$

with $p$ the nucleon momentum, $s$ the nucleon spin, $v=q \cdot p, s^{2}=-m_{N}^{2}, x=Q^{2} / 2 v$, and $Q^{2}=-q^{2}$. The operator product expansion can then be applied to the form factors to extract the leading moments:

$$
\begin{aligned}
2 \int d x x^{n} \mathbf{g}_{1}\left(x, Q^{2}\right) & =\sum_{q=u, d} e_{1, n}^{(q)}\left(\mu^{2} / Q^{2}, g(\mu)\right)\left\langle\mathbf{x}^{\mathbf{n}}\right\rangle_{\Delta \mathbf{q}} \\
2 \int d x x^{n} \mathbf{g}_{2}\left(x, Q^{2}\right) & =\frac{n}{(n+1)} \sum_{q=u, d}\left[2 e_{2, n}^{(q)}\left(\mu^{2} / Q^{2}, g(\mu)\right) \mathbf{d}_{n}^{q}(\mu)+e_{1, n}^{(q)}\left(\mu^{2} / Q^{2}, g(\mu)\right)\left\langle\mathbf{x}^{\mathbf{n}}\right\rangle_{\Delta \mathbf{q}}\right]
\end{aligned}
$$




$$
\begin{aligned}
2 \int d x x^{n-1} \mathbf{F}_{1}\left(x, Q^{2}\right) & =\sum_{q=u, d} c_{1, n}^{(q)}\left(\mu^{2} / Q^{2}, g(\mu)\right)\left\langle x^{n}\right\rangle_{q} \\
\int d x x^{n-2} \mathbf{F}_{2}\left(x, Q^{2}\right) & =\sum_{q=u, d} c_{2, n}^{(q)}\left(\mu^{2} / Q^{2}, g(\mu)\right)\left\langle x^{n}\right\rangle_{q}
\end{aligned}
$$

with Wilson coefficients $e_{1}, e_{2}, c_{1}, c_{2}$ at the energy scale $\mu$ obtained from perturbation calculations, and the structure functions $\left\langle x^{n}\right\rangle_{q},\left\langle x^{n}\right\rangle_{\Delta q}, d_{n}$ calculable as the forward nucleon matrix elements from lattice QCD. We can then use them to reconstruct the form factors $g_{1,2}$ and $F_{1,2}$.
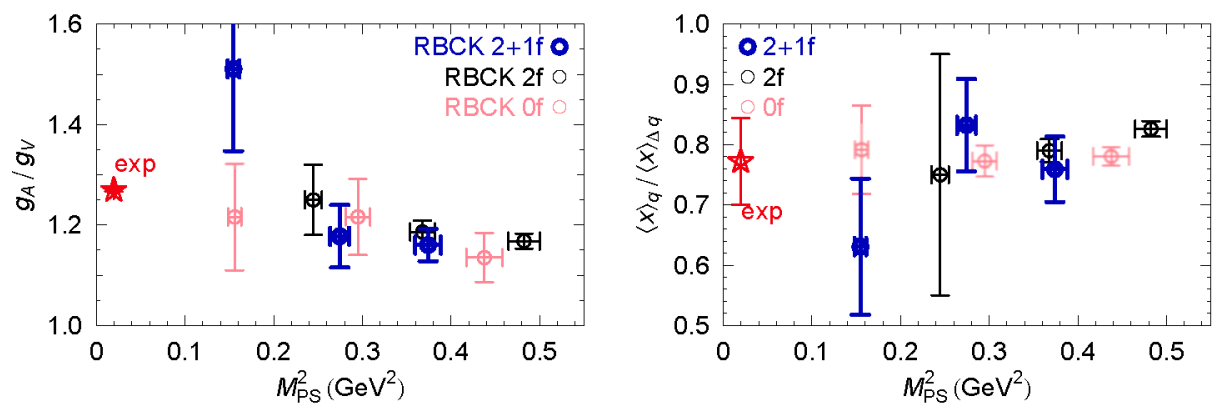

FIGURE 1. Renormalized nucleon axial charge (left) and ratios of first moment (right)

\section{SIMULATION PARAMETERS}

We use domain-wall fermions (DWF) [1] as our sea and valence fermion action because: 1. It has chiral symmetry breaking controlled by the $5^{\text {th }}$-dimensional length $L_{s}$, preventing complicated operator mixing; 2 . The action itself is not only $O(a)$ on-shell improved but also automatically $O(a)$ off-shell improved (due to chiral symmetry); thus one can easily to implement RI/MOM NPR (regularization-independent momentum subtraction scheme nonperturbative renormalization) [5] without extra $O(a)$ off-shell improvement on the action or operators. Some previous work using the quenched approximation can be found in Ref. [2].

The gauge action has been selected from $O\left(a^{2}\right)$-improved gauge actions, with the general form of

$$
S_{g}=\frac{\beta}{3} \operatorname{Re} \operatorname{Tr}\left(\left(1-8 c_{1}\right)\left\langle\mathbf{1}-U_{\text {plaq }}\right\rangle+c_{1}\left\langle\mathbf{1}-U_{\text {rectangle }}\right\rangle\right),
$$

where the constraint $c_{0}+8 c_{1}=1$ is needed to obtain the correct continuum limit. In the 2-flavor case, the RBC collaboration generated lattice configurations using "Doubly blocked Wilson 2" (DBW2) with $c_{1}=-1.40686$ to get smaller chiral symmetry breaking[3]. The ensemble is at lattice spacing $a^{-1} \approx 1.6 \mathrm{GeV}$ (set by Sommer scale $0.5 \mathrm{fm}$ ), with one volume, $\approx 2^{3} \mathrm{fm}^{3}$. The up and down sea quark mass in terms of the inverse lattice spacing are $0.02,0.03$ and 0.04 with the strange quark mass set to 0.04 (near the physical strange quark mass). The pseudoscalar mass at the unitary points $\left(m_{\mathrm{sea}}=m_{\mathrm{val}}\right)$ are $495(4), 607(4)$ and $695(4) \mathrm{MeV}$ respectively. In the 2+1 flavor case[4], we use Iwasaki action with $c_{1}=-0.331$, which has better topological properties than DBW2. The RBC and UKQCD collaborations have generated $2+1$ flavor dynamical DWF ensembles at fixed lattice spacing $a^{-1} \approx 1.6 \mathrm{GeV}$ (set by Sommer scale $0.5 \mathrm{fm}$ ) 
with two volumes, $\approx 2^{3}$ and $3^{3} \mathrm{fm}^{3}$, using Iwasaki gauge action $(\beta=2.13)$. The up and down sea quark mass in terms of the inverse lattice spacing are $0.01,0.02$ and 0.03 with the strange quark mass set to 0.04 and the pseudoscalar mass at the unitary points $\left(m_{\mathrm{sea}}=m_{\mathrm{val}}\right)$ are $393(4), 523.8(2.9), 611.7(2.6) \mathrm{MeV}$ respectively. In both ensembles, we use a Gaussian-smeared source to improve the signals. On the 2-flavor, $m_{\text {sea }}=0.03,0.04$ ensembles, we calculate 220 configurations with 2 sources separated from their sinks by 10 ; on the $m_{\text {sea }}=0.02$, we have 175 configurations with 3 sources, separation 12 ; on the various $2+1$-flavor ensembles, we have 50-60 configurations with 4 sources, separation 12 .

\section{NUMERICAL RESULTS}

On the lattice, the charges and the structure function moments can be calculated from the ratios of the two-point correlation function

$$
\mathscr{C}_{2 \mathrm{pt}}(\vec{p}, t)=\sum_{\alpha, \beta}\left(\frac{1+\gamma_{4}}{2}\right)_{\alpha \beta}\left\langle J_{\beta}(\vec{p}, t) \bar{J}_{\alpha}(\vec{p}, 0)\right\rangle
$$

and the three-point correlation function

$$
\mathscr{C}_{3 \mathrm{pt}}^{\Gamma, \mathscr{p}}(\vec{p}, t, \tau)=\sum_{\alpha, \beta} \Gamma^{\alpha, \beta}\left\langle J_{\beta}(\vec{p}, t) \mathscr{O}(\tau) \bar{J}_{\alpha}(\vec{p}, 0)\right\rangle,
$$

with interpolating field $J_{\alpha}(\vec{p}, t)=\sum_{\vec{x}, a, b, c} e^{i \vec{p} \cdot \vec{x}} \varepsilon^{a b c}\left[u_{\alpha}^{T}\left(y_{1}, t\right) C \gamma_{5} d_{b}\left(y_{2}, t\right)\right] u_{c, \alpha}\left(y_{3}, t\right) \phi\left(y_{1}-\right.$ $x) \phi\left(y_{2}-x\right) \phi\left(y_{3}-x\right)$, operator of interest $\oslash$ and projection $\Gamma$. The definitions of these will be given as we describe the calculations.

The axial charge is calculated with $\mathscr{O}=\bar{q} \gamma_{5} \gamma_{k} q$ and $\Gamma=\frac{1+\gamma_{4}}{2} i \gamma_{5} \gamma_{3}$ in Eq. 6. The renormalized nucleon axial charge is obtained from $\left(g_{A}\right)^{\text {ren }}=g_{A} / g_{V}$ because the local current renormalization is given by $Z_{A}=Z_{V}=1 / g_{V}$ from chiral symmetry. See the left graph in Figure 1. One can further linearly extrapolate to the the physical pion mass to get $\left(g_{A}\right)^{\text {ren }}=1.27(5)$ in the $2 \mathrm{f}$ case and $\left(g_{A}\right)^{\text {ren }}=1.4(2)$ in the $2+1 \mathrm{f}$ case.
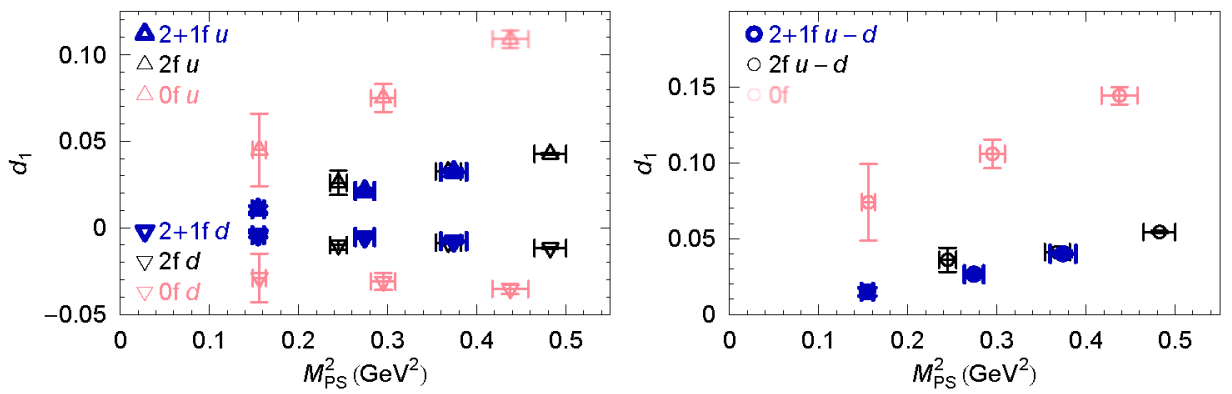

FIGURE 2. Twist-3 matrix elements, $u$ - and $d$-components (left) and isovector contribution(right)

The first moments, $\langle x\rangle_{q}$ and $\langle x\rangle_{\Delta q}$, of the structure functions are $\mathscr{O}_{44}^{q}=$ $\bar{q}\left[\gamma_{4} \overleftrightarrow{D_{4}}-\frac{1}{3} \Sigma_{k} \gamma_{k} \overleftrightarrow{D_{k}}\right] q$ and $\vartheta_{\{34\}}^{5 q}=i \bar{q} \gamma_{5}\left[\gamma_{3} \overleftrightarrow{D_{4}}+\gamma_{4} \stackrel{\leftrightarrow}{D_{3}}\right] q$, which belong to the $\mathbf{3}_{1}^{+}$ and $\mathbf{6}_{3}^{-}$representations respectively. Their values can be extracted from the ratios of

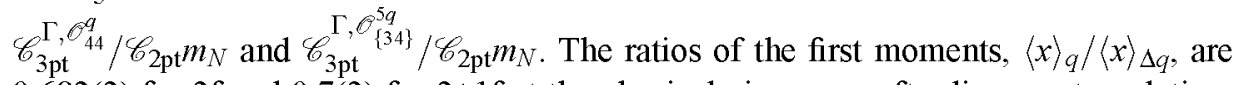
$0.682(2)$ for $2 \mathrm{f}$ and $0.7(2)$ for $2+1 \mathrm{f}$ at the physical pion mass after linear extrapolation. See the right graph in Figure 1. 
The twist-3 matrix element, $d_{1}$, is obtained from $\mathscr{O}_{[34]}^{5 q}=i \bar{q} \gamma_{5}\left[\gamma_{3} \stackrel{\leftrightarrow}{D_{4}}-\gamma_{4} \stackrel{\leftrightarrow}{D_{3}}\right] q$, calculated from $\mathscr{C}_{3 \mathrm{pt}}^{\Gamma, \mathscr{O}^{5 q} q} / \mathscr{C}_{2 \mathrm{pt}}$. The unrenormalized $d_{1}^{\text {bare }}$ is shown in Figure 2 on the right, along with the $u$ - and $d$-components plotted separately (on the left). We got $d_{1}^{\text {bare }}=0.001(2)$ in the $2 \mathrm{f}$ case and $d_{1}^{\text {bare }}=-0.0003(4)$ in the $2+1 \mathrm{f}$ case in the physical pion limit, both of which are consistent with zero.
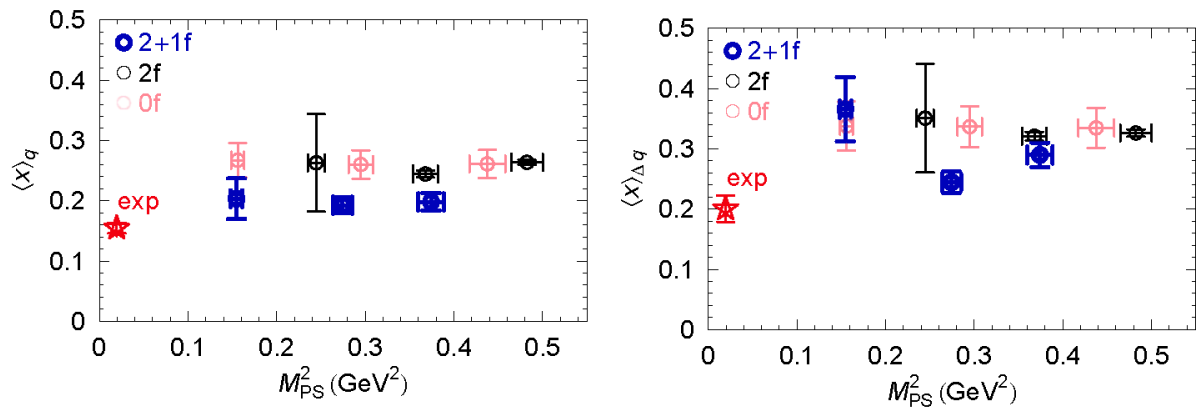

FIGURE 3. Renormalized first moments: $\langle x\rangle_{q}$ (left) and $\langle x\rangle_{\Delta q}$ (right)

We work out the nonperturbative renormalization in RI/MOM scheme to renormalize the above quantities. In general, the operators of interest can mix with lower-dimension operators as $\mathscr{O}_{i}(\mu)=Z_{i}(\mu, a) \mathscr{O}_{i}(\mu)+\sum_{i \neq j} Z_{i j}(\mu ; a) \mathscr{O}_{j}(\mu)$. With the good chiral symmetry of DWF action, however, we are protected from this mixing problem. We calculate $Z_{O_{\Gamma}}(\mu ; a)$ in RI/MOM scheme[5], where $\mu$ must fall inside the renormalization window $\Lambda_{\mathrm{QCD}} \ll \mu \ll 1 / a$. Then we convert to $\overline{\mathrm{MS}}$ scheme, running to $2 \mathrm{GeV}$. Finally, we get the renormalized moments as shown in Figure 3.

\section{CONCLUSION AND OUTLOOK}

In this work, we report on lattice calculations from chiral fermions (DWF) for both sea and valence quarks in 2-flavor and 2+1-flavor dynamical simulations. Our dynamical points line up nicely in the pion mass range $400-700 \mathrm{MeV}$. Our ratios for $g_{A} / g_{V}$ and $\langle x\rangle_{q} /\langle x\rangle_{\Delta q}$ are consistent with the experimental values. Our preliminary renormalized $\langle x\rangle_{\Delta q}$ is up to $50 \%$ higher than the experimental values, but the renormalized $\langle x\rangle_{q}$ is improved in the $2+1$ flavor case. We did not observe any significant curvature in these quantities as a function of quark mass. Our bare twist-3 matrix elements are consistent with zero. It is interesting to note that we also see significant disagreement between quenched and dynamical results for this quantities. We are working on producing greater statistics from our $2+1$ flavor lattices.

This work was done on the QCDSP and QCDOC computers with resources provided by Columbia University, Brookhaven National Laboratory and RIKEN.

\section{REFERENCES}

1. D. B. Kaplan, Phys. Lett. B 288, 342 (1992); Y. Shamir, Nucl. Phys. B 406, 90 (1993).

2. K. Orginos et al., Phys. Rev. D 73, 094503 (2006); S. Sasakiet al., Phys. Rev. D 68, 054509 (2003);

3. Y. Aoki et al., Phys. Rev. D 72, 114505 (2005).

4. D. Antonio et al., RBC and UKQCD collaborations, in preparation.

5. P. Marenzoni, G. Martinelli and N. Stella, Nucl. Phys. B 455, 339 (1995). 